\title{
Alteraciones del sueño en pacientes con enfermedad renal crónica avanzada
}

\author{
Ana Moreno-Egea ${ }^{1}$, Mónica Romero-Reyes ${ }^{1}$, M $^{\mathrm{a}}$ Victoria Gómez-López ${ }^{2}$, Rodolfo Crespo-Montero ${ }^{1,2,3}$ \\ ${ }^{1}$ Departamento de Enfermería. Facultad de Medicina y Enfermería. Universidad de Córdoba. Córdoba. España \\ ${ }^{2}$ Servicio de Nefrología. Hospital Universitario Reina Sofía de Córdoba. Córdoba. España \\ ${ }^{3}$ Instituto Maimónides de Investigación Biomédica de Córdoba. Córdoba. España
}

Como citar este artículo:

Moreno-Egea A, Romero-Reyes M, Gómez-López MV, Crespo-Montero R. Alteraciones del sueño en pacientes con enfermedad renal crónica avanzada.

Enferm Nefrol. 2020 Jul-Sep;23(3):259-266

\section{Resumen}

Introducción: Los pacientes con Enfermedad Renal Crónica Avanzada, sufren una amplia variedad de síntomas físicos y psicológicos. Al llegar a estadios finales, sufren alteraciones significativas en la salud física, mental, emocional y social, destacándose el impacto en la calidad del sueño.

Objetivo: Analizar las principales alteraciones del sueño en pacientes con Enfermedad Renal Crónica Avanzada.

Material y Método: Se llevó a cabo un estudio observacional descriptivo transversal en el Servicio de Nefrología del Hospital Universitario Reina Sofía de Córdoba en pacientes con Enfermedad Renal Crónica Avanzada en estadios 4-5. Se estudiaron las alteraciones del sueño mediante el cuestionario Índice de Calidad de Sueño de Pittsburg y su relación con otras variables como la edad, sexo, comorbilidad, Hemoglobina, Creatinina Sérica e Índice de Masa Corporal.

Resultados: Se estudiaron 119 pacientes, con una edad media de 67,61 $\pm 14,89$ años; 52 mujeres $(43,7 \%)$. El valor medio del Índice de Calidad de Sueño fue de $9,66 \pm 4$ puntos. El $83,2 \%$ de los pacientes presentaban alteraciones del sueño (puntuación $>5$ puntos). Esta

\section{Correspondencia:}

Ana Moreno Egea

Email: ana.moreno.egea@gmail.com puntuación mostró tener una relación significativa con la comorbilidad y la edad. No se encontraron relaciones entre las alteraciones del sueño con el sexo, aclaramiento renal ni Índice de Masa Corporal.

Conclusión: Una mayoría de los pacientes con Enfermedad Renal Crónica Avanzada en estadios 4-5 presentan alteraciones del sueño. Estas alteraciones están relacionadas con mayor comorbilidad y edad avanzada, sin que el sexo, el aclaramiento renal o el Índice de Masa Corporal tengan relación con esta alteración, al menos en la muestra estudiada.

PALABRAS CLAVE: ERCA; prediálisis; trastornos del sueño; insomnio; calidad del sueño.

\section{Sleep disturbances in patients with advanced chronic kidney disease}

\section{Abstract}

Introduction: Patients with advanced chronic kidney disease suffer from a wide variety of physical and psychological symptoms. In final stages, they suffer significant alterations in physical, mental, emotional and social health, highlighting the impact on the quality of sleep.

Objective: To analyse the main sleep disorders in patients with Advanced Chronic Kidney Disease (ACKD). 
Material and Methods: A cross-sectional descriptive observational study was carried out in the Nephrology Unit of the Reina Sofía University Hospital (Córdoba) in patients with ACKD in stages 4-5. Sleep disturbances were studied using the Pittsburg Sleep Quality Index questionnaire as well as the relationship with other variables such as age, sex, comorbidity, Haemoglobin, Serum Creatinine, and Body Mass Index.

Results: 119 patients were studied, with a mean age of $67.61 \pm 14.89$ years; 52 women $(43.7 \%)$. The mean value of the Sleep Quality Index was $9.66 \pm 4$ points. $83.2 \%$ of the patients had sleep disturbances (score> 5 points). A significant relationship was found with comorbidity and age. No relationships were found between sleep disturbances with sex, renal clearance or Body Mass Index.

Conclusion: A large percentage of patients with ACKD in stages 4-5 have sleep disturbances. These alterations are related to greater comorbidity and advanced age; sex, renal clearance or Body Mass Index have not shown a relationship, at least in the sample studied.

KEYWORDS: ACKD; predialysis; sleep disorders; insomnia; quality of sleep.

\section{Introducción}

La enfermedad renal crónica (ERC) es un problema de salud pública con un incremento progresivo de la prevalencia debido principalmente a un envejecimiento de la población, con una importante morbimortalidad asociada y tratamientos de elevado coste económico ${ }^{1,2}$. La enfermedad renal crónica avanzada (ERCA) incluye los estadios 4 y 5 de la clasificación de la ERC, definiéndose como un descenso grave del filtrado glomerular (FG $\left.<30 \mathrm{ml} / \mathrm{minl}, 73 \mathrm{~m}^{2}\right)^{3}$.

En España, en el estudio EPIRCE se estimó que aproximadamente el $10 \%$ de la población adulta presentaba algún grado de ERC, siendo del 6,8\% para los estadios 3-5, aunque existían diferencias importantes con la edad (3,3\% entre $40-64$ años y $21,4 \%$ para mayores de 64 años), y del 0,3\% de ERCA [estadios 4 y 5 ]) 4 .

En esta fase de la enfermedad (estadios 4-5), la sintomatología se hace más evidente, necesitando la mayoría de los pacientes al final del estadio 5, un tratamiento renal sustitutivo ${ }^{5}$, con gran impacto sobre la calidad de vida $^{6,7}$. En este sentido, las alteraciones en la calidad de vida relacionada con la salud aumentan exponencialmente en estas etapas de la ERCA, observándose alteraciones significativas en la salud física, mental, emocional y social, destacándose el impacto en la calidad del sueño $0^{8,9}$.

El sueño es una necesidad biológica que permite que sucedan modificaciones metabólicas, hormonales y bioquímicas necesarias para el buen funcionamiento del organismo. En los pacientes con ERC los problemas relacionados con el sueño se consideran de importancia clínica, ya que, además de afectar negativamente a la calidad de vida, influyen generando estrés, alterando las Actividades Básicas de la Vida Diaria 0, incluso, agravando enfermedades tales como enfermedad cardiovascular crónica ${ }^{10}$.

La prevalencia de los trastornos del sueño en pacientes en con ERCA en estadios 4-5 es del 14 al 85\%, siendo los más frecuentes el síndrome de apnea obstructiva del sueño, insomnio, síndrome de piernas inquietas y somnolencia diurna?.

El síndrome de apnea obstructiva del sueño es una alteración crónica grave provocada por el colapso de las vías aéreas superiores. Las personas con ERCA tienen mayor riesgo de presentar este síndrome al tener factores de riesgo como sobrecarga de volumen o edad avanzada. Estos factores también pueden influir en el insomnio, definido como una latencia mayor a $30 \mathrm{mi}-$ nutos $y / 0$ dos o más despertares nocturnos $\mathrm{y} / 0$ vigilia nocturna mayor a una hora y/o tiempo de sueño total menor a 6 horas, que afecta gravemente en la calidad de vida del paciente. El síndrome de piernas inquietas es una alteración neurosensitiva-motora asociada al sueño, que afecta esencialmente a las piernas. Pese a que su etiología es desconocida, factores como déficit de hierro o alteración en el metabolismo, ambos frecuentes en pacientes con ERC, hace suponer que exista una relación. Por último, la somnolencia diurna es la tendencia de la persona a quedarse dormida durante la vigilia, y puede presentarse como manifestación de cualquiera de las patologías mencionadas anteriormente ${ }^{10,11}$.

Todos estos trastornos del sueño han sido ampliamente estudiados en pacientes con ERCA sometidos a terapia renal sustitutiva renal, siendo numerosos los investigadores que confirman su alta prevalencia ${ }^{11-13}$. Sin embargo, en la etapa de prediálisis existen menos estudios al respecto, algunos autores han comparado la prevalencia de las alteraciones del sueño en pa- 
cientes con ERCA en prediálisis frente a pacientes en diálisis y no han encontrado diferencias entre ambos grupos $^{14}$.

Algunos estudios han encontrado mala calidad del sueño en una muestra amplia en pacientes en la etapa de prediálisis $^{15}$, con alta carga de trastornos del sueño, especialmente en mujeres; y otros autores han observado que la calidad del sueño y la duración del mismo, se asocian a la progresión de la ERC ${ }^{16}$.

Por todo ello, parece relevante el estudio de las alteraciones del sueño en estos pacientes, dado que según ha observado algún autor, van apareciendo a medida que la función renal empeora, aunque no hay mucha bibliografía al respecto; por lo que creemos interesante realizar un análisis sobre las principales alteraciones del sueño que sufren los pacientes con ERCA en estadios avanzados. Por consiguiente el objetivo del presente estudio, fue analizar las principales alteraciones del sueño en pacientes con ERCA en prediálisis.

\section{Material y Método}

\section{Ámbito de estudio}

El estudio se realizó en el Servicio de Nefrología del Hospital Universitario Reina Sofía de Córdoba (HURS). El periodo en el que se desarrolló el estudio fue entre los meses de marzo y mayo de 2020.

\section{Diseño del estudio}

Estudio observacional descriptivo transversal.

\section{Población y muestra}

- Selección de los participantes: la población de estudio fueron los pacientes con ERCA que son atendidos en la consulta de Bajo Aclaramiento del Servicio de Nefrología del HURS.

- Criterios de inclusión: pacientes con ERCA que se encuentren en estadios 4-5 o prediálisis, sin alteraciones cognitivas que le impidan responder a los cuestionarios.

- Tipo de muestreo: se llevó a cabo un muestreo no probabilístico accidental.

\section{Definición de variables}

Como variable de resultado, las alteraciones del sueño. Se han estudiado además otras variables como edad, sexo, filtrado glomerular (FG), Índice de Masa de Corporal (IMC), Creatinina Sérica, Hemoglobina ( $\mathrm{Hgb})$ y comorbilidad.
- Instrumentos de medida: para establecer las principales alteraciones del sueño se utilizó el Índice de Calidad de Sueño de Pittsburg (ICSP) ${ }^{17}$.

Este instrumento consta de 19 preguntas autoaplicadas y de 5 preguntas evaluadas por la pareja del paciente o por su compañero/a (si este está presente) referidas al último mes. Los 19 ítems autoaplicados forman 7 componentes de puntuación, cada uno de los cuales, contiene una puntuación de 0 a 3 puntos, siendo 0 dificultad nula y 3 dificultad severa. Los 7 componentes tienen que sumarse para obtener una puntuación global comprendida entre 0 a 21 puntos, significando 0 puntos no existencia de dificultades y 21 puntos graves dificultades. Una puntuación mayor de 5 puntos muestra que existen alteraciones del sueño.

Para el análisis de la comorbilidad se utilizó el Índice de Comorbilidad de Charlson ajustado por edad $(\mathrm{ICC})^{18}$.

\section{Recogida de datos}

Los datos se iban a recoger en la propia consulta de Bajo Aclaramiento del Servicio de Nefrología, en función de las citas programadas de los pacientes en esta consulta. Ante la situación extraordinaria por el COVID-19, los datos tuvieron que ser obtenidos mediante llamada telefónica. En todo momento, se preservó la confidencialidad de los datos del paciente. La entrevista se realizó a aquellos pacientes que, tras ser informados del estudio y lectura del consentimiento informado, aceptaron participar en este, haciéndoles hincapié en que podían abandonar el mismo cuando lo deseasen. Las llamadas telefónicas se realizaron en días laborables y en horario que no ocasionase molestias al paciente.

El resto de los datos analizados fueron extraídos de la base de datos del Servicio de Nefrología e incorporados en la base de datos de Excel para su análisis.

\section{Análisis estadístico}

Para las variables cualitativas se utilizó una distribución de frecuencias, mientras que para las variables numéricas se usó la media \pm la desviación estándar, o la mediana y el rango intercuartílico, según seguían o no una distribución normal. Para establecer comparaciones entre ambos sexos, se utilizó la t de Student para muestras independientes o la U de Mann Whitney, según siguieron o no una distribución normal. Se realizaron correlaciones con el test de Pearson, para establecer asociaciones entre variables. Se aceptó significación estadística para $\mathrm{p}<0,05$. 


\section{Aspectos éticos y legales}

La información obtenida ha sido tratada conforme a la actual Ley Orgánica 3/2018, de 5 de diciembre, de "Protección de Datos Personales y garantía de los derechos digitales", y con el único fin del desarrollo de esta investigación. La participación en el estudio fue en todo momento de carácter voluntaria y anónima, respetando en cualquier punto de su desarrollo que cualquier usuario decidiera abandonar la entrevista, sin necesidad de justificar el motivo por el cual deja de participar.

El estudio contó con el informe favorable del Comité de Ética e Investigación del HURS (Código TFG-ASPD-2019).

\section{Resultados}

Se estudiaron 119 pacientes con una media de edad de $67,61 \pm 14,89$ años; 67 hombres $(56,3 \%)$ y 52 mujeres $(43,7 \%)$.

En la Tabla 1 se representan los valores de los Componentes del ICSP, con los Intervalos de Confianza de la media al 95\%.

Tabla 1. Valores medios de los componentes del cuestionario ICSP.

\begin{tabular}{lll}
\hline Componentes (ICSP) & Media \pm DE & IC al 95\% \\
\hline Calidad del sueño & $1,32 \pm 0,62$ & $1,21-1,43$ \\
\hline Latencia del sueño & $1,55 \pm 1,02$ & $1,36-1,73$ \\
\hline Duración del sueño & $1,18 \pm 0,83$ & $1,03-1,34$ \\
\hline Eficiencia del sueño & $1,57 \pm 0,92$ & $1,40-1,74$ \\
\hline Perturbaciones del sueño & $1,56 \pm 0,54$ & $1,46-1,66$ \\
\hline Uso de medicación hipnótica & $1,03 \pm 1,39$ & $0,77-1,28$ \\
\hline Disfunción diurna & $1,47 \pm 0,71$ & $1,34-1,60$ \\
\hline Puntuación Total & $9,68 \pm 4,01$ & \\
\hline
\end{tabular}

Tabla 2. Valores medios de las características clínicas estudiadas.

\begin{tabular}{lcc}
\hline & Media \pm DE & IC al 95\% \\
\hline ICC (puntos) & $6,08 \pm 2,4$ & $5,52-6,54$ \\
\hline FG $\left(\mathrm{ml} / \mathrm{min} / 1,73 \mathrm{~m}^{2}\right)$ & $18,83 \pm 5,87$ & $17,16-19,39$ \\
\hline Creatinina sérica $(\mathrm{mg} / \mathrm{dl})$ & $3,41 \pm 0,88$ & $3,25-3,57$ \\
Hgb $(\mathrm{gr} / \mathrm{dl})$ & $11,87 \pm 1,65$ & $11,60-12,27$ \\
IMC $\left(\mathrm{Kg} /[\mathrm{metros}]^{2}\right)$ & $29,8 \pm 5,6$ & $28,35-30,88$ \\
\hline
\end{tabular}

ICM:Índice de Comorbilidad de Charlson. FG: Filtrado Glomerular. Hgb: Hemoglobina. IMC: Índice de Masa Corporal.

incluyeron en el estadio 4 a los pacientes con un FG entre 15 y $30 \mathrm{ml} / \mathrm{min} / 1,73 \mathrm{~m}^{2}$, y en estadio 5 a pacientes con $\mathrm{FG}<15 \mathrm{ml} / \mathrm{min} / 1,73 \mathrm{~m}^{2}$. En estadio 4 se encontraron 37 pacientes $(31,1 \%)$ y en estadio 5,82 pacientes $(68,9 \%)$.

Al comparar las distintas variables analizadas entre los pacientes en estadio 4 y estadio 5, no se encontraron diferencias significativas, en ninguna de las variables de estudio.

Utilizando el punto de corte de 5 en la Puntuación Total del PSQI, se dividió a los pacientes en 2 grupos: pacientes con $<5$ puntos a los que denominamos Sin alteración del sueño y pacientes con $>5$ puntos a los que denominamos Con alteración del sueño.

Sin alteraciones del sueño resultaron 20 pacientes (17\%) y con alteraciones del sueño 99 pacientes ( $83 \%$ ).

Cuando se compararon los valores de las distintas variables, tan solo se encontraron diferencias estadísticamente significativas en la edad y el ICC, entre ambos grupos, tal como puede apreciarse en la Tabla 3.

ICSP: Índice de Calidad del Sueño de Pittsburgh

En la Tabla 2 se representan los valores medios de las características clínicas estudiadas (ICC, FG, creatinina sérica, Hgb e IMC).

La Puntuación Total de la Calidad del Sueño del ICSP se correlacionó estadísticamente con la edad $(r=0,211 ; p<0,05)$ y el ICC $(r=0,224 ; p<0,05)$.

Para analizar la influencia del FG en las distintas variables se dividió a los pacientes de acuerdo a los estadios 4 y 5 de la ERCA. Se
Tabla 3. Comparación entre pacientes "Sin alteraciones del sueño" y pacientes "Con alteraciones del sueño, para las distintas variables.

\begin{tabular}{|lccc}
\hline & $\begin{array}{c}\text { Sin alteración } \\
\text { del sueño }\end{array}$ & $\begin{array}{c}\text { Con alteración } \\
\text { del sueño }\end{array}$ & Valor de p \\
\hline ICC (puntos) & $5,00 \pm 2,26$ & $6,28 \pm 2,39$ & $\mathrm{p}<0,05$ \\
\hline Creatinina sérica $(\mathrm{mg} / \mathrm{dl})$ & $3,7 \pm 0,9$ & $3,3 \pm 0,8$ & $\mathrm{NS}$ \\
\hline Hgb (gr/dl) & $12,46 \pm 2,04$ & $11,75 \pm 1,54$ & $\mathrm{NS}$ \\
\hline FG (ml/min/ $\left.1,73 \mathrm{~m}^{2}\right)$ & $18,2 \pm 5$ & $18,9 \pm 5$ & $\mathrm{NS}$ \\
\hline IMC (Kg/estatura $\left.\left[\mathrm{mt}^{2}\right]^{2}\right)$ & $29,01 \pm 5,28$ & $29,76 \pm 6,92$ & $\mathrm{NS}$ \\
\hline Edad (años) & $61,50 \pm 18,53$ & $68,84 \pm 13,82$ & $\mathrm{p}<0,05$ \\
\hline
\end{tabular}

ICC: Índice de Comorbilidad de Charlson. FG: Filtrado Glomerular. Hgb: Hemoglobina. IMC: Índice de Masa Corporal. 
Respecto al sexo, la puntuación media de las mujeres para el ICSP fue $9,8 \pm 4,4$ frente $9,4 \pm 3,9$ de los hombres (NS).

Respecto a otras relaciones estadísticas, se encontró relación del ICC con la edad, $(r=0,559 ; p<0,01)$, con la Creatinina sérica $(r=-0,186 ; p<0,05)$ y la $\mathrm{Hgb}(r=-$ $0,209 ; p<0,05)$.

En cuanto a las percepciones de los pacientes, según los diferentes componentes, podemos destacar que en el componente 1 , el 66,4\% manifestaron tener una $\mathrm{Ca}$ lidad del sueño subjetiva Buena y el 25,2\% Mala. En el componente 2, Latencia del sueño o tiempo que tardaban en dormirse, el $37 \%$ de los pacientes manifestaron entre $16-30$ minutos y el $23 \%$ entre $31-60$ minutos. En el componente 3, Duración del sueño, el 53\% manifestaron dormir entre 6-7 horas y el $20 \%$ entre 5-6 horas. En el componente 4, Eficiencia del sueño habitual ( $\left[n^{\circ}\right.$ de horas dormidas $/ n^{\circ}$ de horas permanecidas en la cama]+100), el $45 \%$ de los pacientes tenían una eficiencia del sueño entre $75-84 \%$ y el $24,5 \%$ una eficiencia entre $65-74 \%$. En el componente 5, Perturbaciones del sueño, el 53,8\% tenía 1 o veces perturbaciones a la semana y el $44,7 \%$ tenía menos de 1 perturbación a la semana. En el componente 6, Uso de medicamentos para dormir, el 63,9\% dijo no tomar ninguna medicación en el último mes y el $31 \%$ dijo tomar 3 o más veces medicación para dormir a la semana. Por último en el componente 7, Disfunción diurna (somnolencia mientras conducía, comía o desarrollando otras actividades, el $50,4 \%$ de los pacientes manifestaron que menos de 1 vez a la semana y el $37 \%$ una o 2 veces a la semana.

\section{Discusión}

Los pacientes con ERCA sufren una amplia variedad de síntomas físicos y psicológicos, siendo las alteraciones del sueño frecuentes en este grupo ${ }^{9}$. Algunos autores han investigado sobre las alteraciones del sueño en pacientes con ERCA sometidos a TSR ${ }^{14}$, pero no hay suficiente evidencia en paciente en prediálisis, de ahí que este fuese nuestro objetivo.

La prevalencia de pacientes con mala calidad del sueño (ICSP superior a 5 puntos) fue de $83 \%$. Este dato es similar al de estudios anteriores, que estiman la prevalencia de pacientes con ERCA con mala calidad del sueño, entre el 14 y $85 \%{ }^{19,20}$. En nuestro estudio, la media del ICSP para los hombres fue de $9,8 \pm 4$ y para las mujeres $9,4 \pm 3$; no existiendo, por tanto diferencias entre sexos. La posible influencia del sexo en las alteraciones del sueño es un tema controvertido en la literatura de pacientes con ERCA. Algunos autores afirman que no hay diferencias significativas, pero otros afirman que la prevalencia es mayor en mujeres que en hombres, como el estudio realizado por Sekercioglu N, que además plantea la posibilidad de que la prevalencia sea mayor conforme aumenta la edad ${ }^{16}$.

La media de edad de la población fue de $67,61 \pm 14,89$ años, encontrándose una relación significativa entre esta y las alteraciones del sueño en el total de la muestra, en la misma línea del estudio de Zhang, J, que observa que la edad avanzada es uno de los factores que más influye en la prevalencia de problemas del sueño ${ }^{15}$. Aunque otros autores, no encontraron relación entre la edad y las alteraciones del sueño ${ }^{16}$; lo cual ocurre también en pacientes con terapia renal sustitutiva, con estudios que encuentran relación entre la edad y las alteraciones del sueño y otros que no la encuentran ${ }^{21,22}$. Esta contradicción con respecto a la edad puede deberse a diferencias de la población de estudio y a las herramientas de medición. El correcto diagnóstico para las alteraciones del sueño es una polisomnografía, y la mayoría de los estudios, no utilizan esta herramienta ${ }^{19}$.

La posible relación estadística entre la puntuación del ICSP y otras variables como la $\mathrm{Hgb}, \mathrm{FG}$ o Creatinina sérica, es otro aspecto controvertido en pacientes con ERCA. En nuestro estudio, no encontramos relación con ninguno de estos datos de laboratorio, con lo cual coincidimos con otros autores como Zhang J., que sugiere que la mala calidad del sueño puede existir en una etapa muy temprana de la ERC y que la disminución de la función renal y otras variables de laboratorio específicas podrían no ser la causa directa de la falta de sueño en la $E C^{15,23}$. No obstante, hay estudios que sí observan una fuerte relación entre $F G$ y la calidad del sueño en pacientes con ERCA ${ }^{19}$.

EI IMC constituye uno de los principales factores de riesgo para LA ERCA. La media de IMC tanto para el grupo de pacientes sin alteración del sueño, como con alteración del sueño, se encuentra en el rango de Obesidad. Algunos autores que han estudiado la relación entre obesidad y calidad de vida en pacientes con ERCA, destacan que la obesidad influye considerablemente en la calidad del sueño de estos pacientes ${ }^{24}$, así como la relación entre el IMC con el ICC ${ }^{25}$.

La media de la puntuación del ICC para el total de la muestra es alta $(6,08 \pm 2,40)$, en la línea de estudios 
anteriores $^{7}$. Además, se relaciona significativamente con las alteraciones del sueño, es decir, a mayor comorbilidad más alteraciones en el sueño en el total de la muestra estudiada.

Como aspectos más reseñables de las características de las alteraciones del sueño presentadas por los pacientes, destacar que el $81,5 \%$ tienen una duración del sueño inadecuada. Dormir menos de 7 horas se asocia a mayor riesgo de complicaciones, como hipertensión arterial, diabetes mellitus tipo 2 y enfermedades coronarias, todas ellas factores de riesgo para ERCA ${ }^{26}$. Otro aspecto destacable es que el $90 \%$ de los pacientes tenían un porcentaje de eficiencia del sueño menor al $85 \%$ (número de horas de sueño entre número de horas pasadas en la cama $)^{12}$. Estos datos inducen a pensar que la mayor parte de los pacientes estudiados no consiguen tener un sueño satisfactorio, con las implicaciones que ello conlleva.

En cuanto al uso de medicación para dormir (componente 6), el $63,9 \%$ de la muestra no había tomado ninguna en el último mes. El principal fármaco que se utiliza para problemas de sueño en pacientes con ERCA son las benzodiacepinas. Sin embargo, pese a la evidencia de problemas del sueño, la mayor parte no toma medicación, habiéndose señalado que puede estar relacionado con la disminución de la función renal ${ }^{27}$.

Como principales limitaciones de este estudio podemos destacar que la muestra es pequeña debido a las dificultades para que los pacientes quisieran participar en el estudio por teléfono en plena pandemia por el COVID-19, y sobre todo, la imposibilidad de poder entrevistar personalmente a los pacientes en consulta, pues por teléfono algunos mostraban desconfianza con la entrevistadora, por lo que las respuestas podrían haber perdido objetividad.

A la vista de estos resultados podemos concluir que las alteraciones del sueño son un problema importante que afecta a buena parte de los pacientes en estadio 4 y 5 de la ERCA. En nuestra muestra el $83 \%$ de los pacientes padecen alteraciones del sueño.

Estas alteraciones están relacionadas con mayor comorbilidad y tener más edad, sin que el sexo, el aclaramiento renal, la Creatinina sérica, la $\mathrm{Hgb}$ o el IMC tengan relación con esta alteración, al menos en la muestra estudiada.

Pese a que no existen conclusiones claras, parece importante que la calidad del sueño sea tratada lo antes posible para conseguir que no aumente y llegue a afec- tar a la calidad de vida del paciente, pues las alteraciones del sueño parecen ser inherentes a la ERCA desde sus primeros estadios. En este sentido las consultas ERCA de enfermería pueden tener un papel relevante en la detección precoz de esta alteración tan prevalente en estos pacientes.

Recibido: 05-06-20

Revisado: 22-06-20

Modificado: 15-07-20

Aceptado: 15-08-20

\section{Bibliografía}

1. Vanholder R, Annemans L, Brown E, Gansevoort R, Gout-Zwart JJ, Lameire N, et al. European Kidney Health Alliance. Reducing the costs of chronic kidney disease while delivering quality health care: $A$ call to action. Nat Rev Nephrol. 2017;13:393-409.

2. Wang V, Vilme $H$, Maciejewski ML, Boulware LE. The economic burden of chronic kidney disease and end-stage renal disease. Semin Nephrol. 2016; 36:319-30.

3. R. Alcázar-Arroyo, L. Enfermedad Renal Crónica Avanzada. Rev Nefrol. 2008;28(S3):3-6.

4. Otero A, de Francisco A, Gayoso P, García F. Prevalencia de la insuficiencia renal crónica en España: Resultados del estudio EPIRCE. Nefrología. 2010; 30:78-86.

5. Guerrero MA, Toro FJ, Batalha $P$, Salgueira $M$, González F, Marrero S, et al. Estudio ERCA. Características basales, evaluación de la aplicación de la información estructurada para la elección de tratamiento renal sustitutivo y evolución a un año de los pacientes incidentes en la consulta ERCA. Nefrología. 2019;39(6):563-82. 
6. Rebollo-Rubio A, Morales-Asencio JM, Pons-Raventós ME, Mansilla-Francisco JJ. Revisión de estudios sobre calidad de vida relacionada con la salud en la enfermedad renal crónica avanzada en España. Nefrología. 2015;35(1):92-109.

7. Garrido-Blanco R, Arroyo-Priego $E$, Arana-Ruiz AI, López-Zamorano MD, Tierno-Tendero C, Crespo-Montero R. Calidad de vida y enfermedad renal crónica avanzada. Influencia del aclaramiento renal. Enferm Nefrol 2018;21(4):359-67.

8. Sanz-Guajardo D, Orte L, Gómez-Campderá F, Fernández E, Aguilar MD, Lázaro P. Calidad de vida en los pacientes con insuficiencia renal crónica. Influencia de la intervención precoz del nefrólogo y de la consulta prediálisis. Nefrología. 2006;26(3):5665.

9. Gutiérrez D, Leiva J, Macías M, Cuesta A. Perfil sintomático de los pacientes con Enfermedad Renal Crónica Estadio 4 y 5. Enferm Nefrol. 2017; 20(3):256-66.

10. Serrano-Navarro I, Mesa-Abad P, Tevar-Muñoz L, Crespo-Montero R. Trastornos del sueño en el paciente con enfermedad renal crónica avanzada. Enferm Nefrol. 2019; 22(4):361-78.

11. Tenorio Cárcamo G, Barrios Araya S. Alteraciones del sueño más frecuentes en personas en diálisis. Enferm Nefrol. 2018; 21(4):317-33.

12. Restrepo D, Cardeño C. Trastornos de sueño en los pacientes en diálisis. Rev Colomb Psiquiatr. 2010; 39(3):588-600.

13. Restrepo D, Hidalgo P, Gómez C, Gil F, Cardeño C. Trastornos del sueño en pacientes en hemodiálisis. Rev Colomb Psiquiat 2011;40(3):433-45.

14. Shafi S, Shafi T. A comparison of quality of sleep between patients with chronic kidney disease not on hemodialysis and end- stage renal disease on hemodialysis in a developing country. Ren Fail 2017; 39(1):623-28.

15. Zhang J, Wang C, Gong W, Peng H, Tang Y, Cui C et al. Association between sleep quality and cardiovascular damage in predialysis patients with chronic kidney disease. BMC Nephrology. 2014;15,131:19.
16. Sekercioglu N, Curtis B, Murphy S, Barret B. Sleep quality and its correlates in patients with chronic kidney disease: a cross- sectional design. Ren fail. 2015;37(5):757-62.

17. Luna Y, Robles Y, Agüero Y. Validación del Índice de calidad de sueño de Pittsburg en una muestra peruana. Anal Salud Ment 2015;31(2):23-30.

18. Charlson M, Szatrowski TP, Peterson J, Gold J. Validation of a combined comorbidity index. J Clin Epidemiol. 1994;47(11):1245-51.

19. Guney I., Akgul Y, Gencer V, Aydemir H, Asian U, Ecirli S. Sleep Quality and Risk Factors of Atherosclerosis in Predialysis Chronic Kidney Disease. Int J Artif Organs. 2016:39(11):563-69.

20. Su-Ah Sung, Young Youl Hyun, Kyu Beck Lee, Hayne Cho Park, Wookyung Chung, Yeong Hoon Kim, et al. Sleep Duration and Health-Related Quality of Life in Predialysis CKD. Clin J Am Soc Nephrol. 2018; 13(6):858-65.

21. Roumelioti M, Argyropoulos C, Buysse D, Nayar H, Steven W, Unruh M. Sleep Quality, Mood, Alertness and Their Variability in CKD and ESRD. Nephron Clin Pract. 2010;114(4):277-87.

22. Catunda J, Barroso R, Alburquerque $L$, Oswaldo $A$, Gutiérrez A, Carvalhedo F, Meireles V. Sleep disturbances in patients on maintenance hemodialysis: role of dialysis shift. Rev Assoc Med Bras. 2007; 53(6):492-96.

23. Chao-Ying T, Yu-Hsiang C, Yu-Hsuan L, Huang W. Sleep and emotional disturbance in patients with non-dialysis chronic kidney disease. J Formos Med Assoc. 2019;118(6):986-94.

24. Schweitzer ML, Stengel $B$, Legrand $K$, Briançon $S$, Jacquelinet $C$, Combe, $C$,et al. Obesity phenotype and patient- reported outcomes in moderate and severe chronic kidney disease: a cross-sectional study from the CKD-REIN cohort study. Qual Life Res. 2019;28(7):1873-83.

25. Pons Raventos E, Rebollo Rubio A, Amador Coloma R. Utilidad del índice de masa corporal con enfermedad renal crónica. Rev Seden. 2017. 20(4):31622. 
26. Ricardo AC, Knutson K, Chen J, Appel LJ, Bazzano $L$, Carmona-Powell $E$, et al. The Association of Sleep Duration and Quality with CKD Progression. J Am Soc Nephrol. 2017.28(12):3708-15.

27. Lindner AV, Novak M, Bohra M, Mucsi I. Insomnia in Patients With Chronic Kidney Disease. Semin Nephrol. 2015;35(4):359-72.

Este artículo se distribuye bajo una Licencia Creative Commons Atribución-NoComercial 4.0 Internacional. https://creativecommons.org/licenses/by-nc/4.0/

\section{Open Access (c) (7)}

